\title{
Gain of $12 p$ encompassing CCND2 is associated with gemistocytic histology in IDH mutant astrocytomas
}

Felix Sahm ${ }^{1,2}$, Andrey Korshunov ${ }^{2}$, Daniel Schrimpf ${ }^{1,2}$, Damian Stichel ${ }^{1,2}$, David T.W. Jones ${ }^{3}$, David Capper $^{1,2}$, Christian Koelsche ${ }^{1,2}$, David Reuss ${ }^{1,2}$, Annekathrin Kratz ${ }^{1,2}$, Kristin Huang ${ }^{1,2}$, Annika K. Wefers $^{1,2}$, Matthias Schick ${ }^{4}$, Melanie Bewerunge-Hudler ${ }^{4}$, Michel Mittelbronn ${ }^{5}$, Michael Platten ${ }^{6,7,8}$, Daniel Hänggi ${ }^{9}$, Astrid Jeibmann ${ }^{10}$, Andreas Unterberg ${ }^{11}$, Christel Herold-Mende ${ }^{11}$, Stefan M. Pfister $^{3,12}$, Sebastian Brandner ${ }^{13}$, Wolfgang Wick ${ }^{6,14}$, Andreas von Deimling ${ }^{1,2}$

1. Department of Neuropathology, Institute of Pathology, Ruprecht-Karls-University Heidelberg, Heidelberg, Germany,

2. Clinical Cooperation Unit Neuropathology, German Consortium for Translational Cancer Research (DKTK), German Cancer Research Center (DKFZ), Heidelberg, Germany

3. Division of Pediatric Neurooncology, German Consortium for Translational Cancer Research (DKTK), German Cancer Research Center (DKFZ), Heidelberg, Germany

. Genomics and Proteomics Core Facility, Microarray Unit, German Cancer Research Center (DKFZ), Heidelberg, Germany Department of Neuropathology (Edinger Institute), University Hospital Frankfurt, Frankfurt, Germany Neurology Clinic, University Hospital Heidelberg, Heidelberg, Germany

Clinical Cooperation Unit Neuroimmunology and Brain Tumor Immunology, German Consortium for Translational Cancer Research (DKTK), German Cancer Research Center (DKFZ), Heidelberg, Germany Neurology Clinic, University Hospital Mannheim, Mannheim, Germany Department of Neurosurgery, University Hospital Mannheim, Mannheim, Germany Institute of Neuropathology, University Hospital Münster, Münster, Germany

Department of Neurosurgery, University Hospital Heidelberg, Heidelberg, Germany

2. Department of Pediatric Oncology, Haematology and Immunology, Heidelberg University Hospital, and National Center for Tumor Diseases (NCT), Heidelberg, Germany

3. Division of Neuropathology of the UCL Institute of Neurology in London, London, UK

14. Clinical Cooperation Unit Neurooncology, German Consortium for Translational Cancer Research (DKTK), German Cancer Research Center (DKFZ), Heidelberg, Germany

Gemistocytic astrocytoma represents a small subgroup accounting for approximately $5 \%$ of diffuse astrocytic glioma. The WHO classification defines gemistocytic astrocytoma by presence of "a conspicuous, though variable, fraction of gemistocytic neoplastic astrocytes" [5]. These cells should account for at least $20 \%$ of the tumor cells. The term "gemistocytic" was coined by Nissl in 1904 for cells with homogeneous, faintly eosinophilic cytoplasms with short branching processes. It originates from the German word "gemästet" (filled, stuffed, swollen), sometimes also referred back to the Greek "gemistos" with similar meaning.

The neoplastic nature of gemistocytic cells in astrocytoma could be clearly demonstrated by binding of an IDH1R132H mutant protein specific antibody [1]. However, so far no molecular drivers characteristic for this gemistocytic differentiation have been identified. Previous molecular analyses reported higher frequencies of TP53 and PTEN mutations [14], lower frequency of IDH mutations, and alterations in RRAS and ERCC1 [7]. Several studies found shorter progression-free-survival in gemistocytic astrocytoma cases compared to fibrillary astrocytoma [4,8-10], whilst others did not confirm this finding [a reference should be given here].. However, there appears to be a contradiction between the accelerated progression and alower proliferative activity of gemistocytes compared to other tumor cells in the same sample, or in general in diffuse glioma [2-4,15] . To identify potentially recurrent alterations associated with gemistocytic morphology in astrocytoma we performed high-throughput high-resolution genetic and epigenetic analysis on a set of 24 gemistocytic astrocytomas. The control group consisted of $47 \mathrm{IDH}$ mutant astrocytomas WHO grade II, 104 IDH mutant anaplastic astrocytomas WHO grade III, and 293 IDH wild-type glioblastomas WHO grade IV.

The Illumina Infinium HumanMethylation450 BeadChip (450k) array data was used for methylation profiling and to calculate a low-resolution copy number profile (CNP) as previously described [12]. 
Targeted re-sequencing was performed on the genes and with the technology as reported previously [11].

A distinctive feature of tumours with gemistocytic histology was a recurrent numerical aberration in the telomeric region of chromosomal arm 12p, encompassing CCND2 (Figure 1A). An integrated analysis of copy-number variation (Stichel et al., in preparation) in all gemistocytic astrocytoma of our cohort also indicated $12 \mathrm{p}$ as the most consistently altered locus (Figure 1B).

In particular, focal gain of CCND2 and adjacent regions was seen in 8 of 9 gemistocytic astrocytomas WHO grade II and in 13 of 15 anaplastic astrocytomas with distinct gemistocytic morphology (Table 1). Instead, this alteration was observed in only 5 of 47 fibrillary astrocytomas WHO grade II and in only 19 of 104 anaplastic astrocytomas lacking gemistocytic morphology. The alterations detected by analysis of copy number plots based on 450K analysis were confirmed by FISH in a subset of 18 cases (11 gemistocytic cases, 7 fibrillary cases), yielding concordance in 17 out of 18 (94\%) cases (Figure 1B). The single non-concordant case was an anaplastic gemistocytic astrocytoma without indications of chromosome 12 gain by 450k but low-level gain detected by FISH probe directed against 12p12 encompassing CCND2.

This difference was highly significant within grade II and grade III gliomas, respectively (each $p<0.0001$, Fisher's exact test). Also, the event of $12 p / C C N D 2$ gain was significantly associated with gemistocytic histology over the entire diffuse astrocytic glioma cohort $(p<0.0001$, Table 1$)$. Unsupervised clustering of methylome profiles from gemistocytic and fibrillary astrocytoma did not separate these from each other (data not shown).

To assess the mutational landscape of gemistocytic astrocytoma, 17 tumours were further analysed by panel sequencing. All cases harboured IDH1R132 mutations (16/17 IDH1R132H, 1/17 IDH1R132G) and TP53 mutations. Other recurrently mutant genes were ATRX (10/17), ALK (2/17), CSF1R (2/17), FGFR1 (2/17), GSE1 (2/17), MSH6 (2/17), NF1 (2/17), and SMO (2/17, not affecting the activating hotspots). These findings are in line with studies describing high rates of TP53 mutations in gemistocytic astrocytomas, but contrasts reports on high frequencies of PTEN mutations of which none was found in the present set.

Recent reports suggested aberrations of copy number and methylation of ERCC1 and RRAS as a possible marker for gemistocytic astrocytoma [7]. However, we could not detect these aberrations in our dataset (mean beta values for ERCC1 promoter sites 0.09 and 0.1 , for RRAS promoter sites 0.08 and 0.09 , in gemistocytic and control samples, respectively).

Upregulation of CCND2 due to higher copy abundance also provides an explanation for several prior observations on this sub-entity: CCND2 is physiologically upregulated in radial glial cells of the subventricular zone during brain development, and activating CCND2 mutations result in megalencephaly whilst abrogation of CCND2 leads to microcephaly [6]. The higher abundance of CCND2 protein might also disrupt the regular cell cycle, preventing the transition from S to G2 phase, and explain lower mitotic activity but higher pleomorphism with higher number of multi-nucleated cells in such cases. Moreover, the recent approval of inhibitors of the CDK4/6 axis [13], both interacting with CCND2, also opens an additional therapeutic approach for this glioma subtype. 
Table 1 Subtypes of diffuse glioma and $12 p$ status

\section{Subtype}

all All (56)

All gem (9)

All non-gem (47)

all Alll (119)

Alll gem (15)

Alll non-gem (104)

all GBM (293)

12 p gain
$13(23 \%)$
$8(89 \%)$
$5(11 \%)$
$29(24 \%)$
$13(87 \%)$
$19(18 \%)$
$32(11 \%)$

12p balanced/del

$43(77 \%)$

$1(11 \%)$

$42(89 \%)$

$90(76 \%)$

$2(13 \%)$

$85(82 \%)$

$261(89 \%)$

\section{Figure 1}

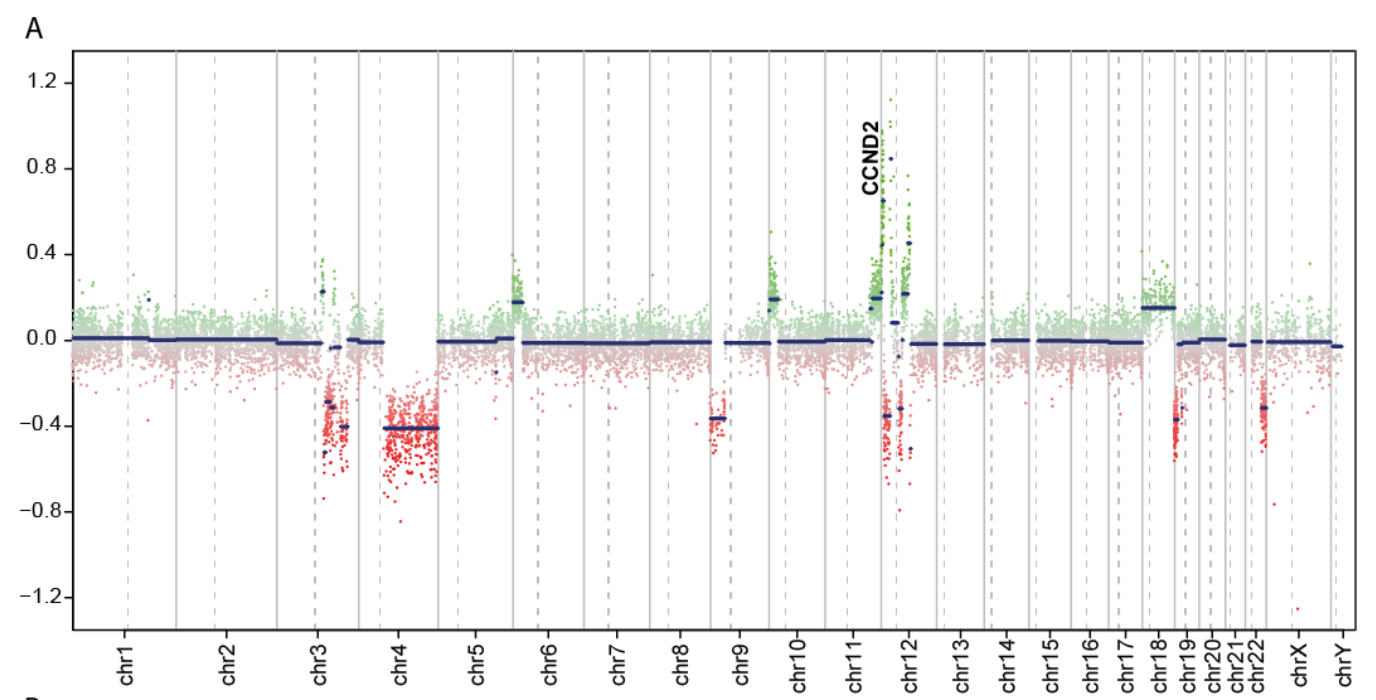

B

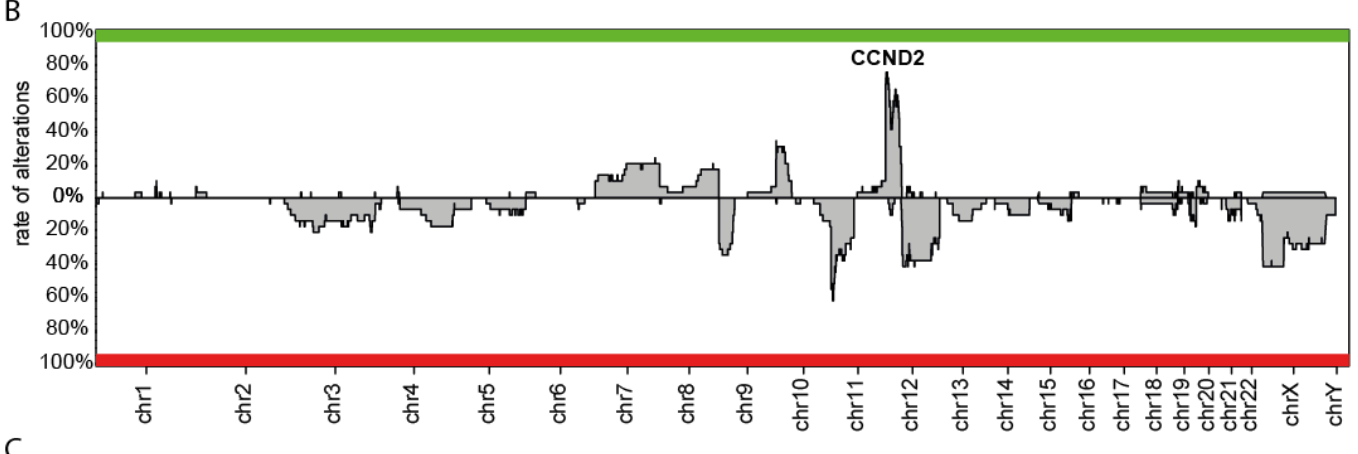

C
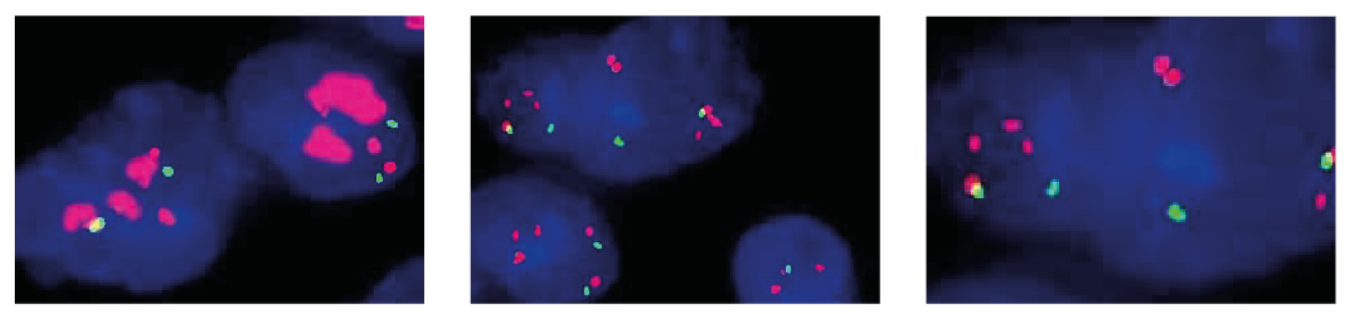

Figure 1 Representative copy-number profile of a gemistocytic astrocytoma (A). Integrated copynumber analysis across all gemistocytic astrocytoma (B). Fluorescence in-situ hybridization of a case with amplification (left) and low-level gain of CCND2 (middle, higher magnification right). 


\section{References}

1 Capper D, Sahm F, Hartmann C, Meyermann R, von Deimling A, Schittenhelm J (2010) Application of mutant IDH1 antibody to differentiate diffuse glioma from nonneoplastic central nervous system lesions and therapy-induced changes. The American journal of surgical pathology 34: 1199-1204

2 Hoshino T, Wilson BC, Ellis WG (1975) Gemistocytic astrocytes in gliomas. An autoradiographic study. Journal of neuropathology and experimental neurology 34: 263-281

3 Kros JM, Schouten WC, Janssen PJ, van der Kwast TH (1996) Proliferation of gemistocytic cells and glial fibrillary acidic protein (GFAP)-positive oligodendroglial cells in gliomas: a MIB-

1/GFAP double labeling study. Acta Neuropathol 91: 99-103

$4 \quad$ Krouwer HG, Davis RL, Silver P, Prados M (1991) Gemistocytic astrocytomas: a reappraisal. Journal of neurosurgery 74: 399-406

5 Louis DN, Perry A, Reifenberger Get al. (2016) The 2016 World Health Organization Classification of Tumors of the Central Nervous System: a summary. Acta Neuropathol 131: 803-820

6 Mirzaa GM, Parry DA, Fry AEet al. (2014) De novo CCND2 mutations leading to stabilization of cyclin D2 cause megalencephaly-polymicrogyria-polydactyly-hydrocephalus syndrome. Nature genetics 46: 510-515

7 Ohta T, Kim YH, Oh JEet al. (2014) Alterations of the RRAS and ERCC1 genes at 19q13 in gemistocytic astrocytomas. Journal of neuropathology and experimental neurology 73: 908915

8 Okamoto Y, Di Patre PL, Burkhard Cet al. (2004) Population-based study on incidence, survival rates, and genetic alterations of low-grade diffuse astrocytomas and oligodendrogliomas. Acta Neuropathol 108: 49-56

9 Peraud A, Ansari H, Bise K, Reulen HJ (1998) Clinical outcome of supratentorial astrocytoma WHO grade II. Acta Neurochir (Wien) 140: 1213-1222

10 Peraud A, Kreth FW, Wiestler OD, Kleihues P, Reulen HJ (2002) Prognostic impact of TP53 mutations and P53 protein overexpression in supratentorial WHO grade II astrocytomas and oligoastrocytomas. Clinical cancer research : an official journal of the American Association for Cancer Research 8: 1117-1124

11 Sahm F, Schrimpf D, Jones DTet al. (2015) Next-generation sequencing in routine brain tumor diagnostics enables an integrated diagnosis and identifies actionable targets. Acta Neuropathol:

12 Sturm D, Witt H, Hovestadt Vet al. (2012) Hotspot mutations in H3F3A and IDH1 define distinct epigenetic and biological subgroups of glioblastoma. Cancer cell 22: 425-437

13 Walker AJ, Wedam S, Amiri-Kordestani Let al. (2016) FDA Approval of Palbociclib in Combination with Fulvestrant for the Treatment of Hormone Receptor-Positive, HER2Negative Metastatic Breast Cancer. Clinical cancer research : an official journal of the American Association for Cancer Research 22: 4968-4972

14 Watanabe K, Peraud A, Gratas C, Wakai S, Kleihues P, Ohgaki H (1998) p53 and PTEN gene mutations in gemistocytic astrocytomas. Acta Neuropathol 95: 559-564

15 Watanabe K, Tachibana O, Yonekawa Y, Kleihues P, Ohgaki H (1997) Role of gemistocytes in astrocytoma progression. Lab Invest 76: 277-284 